\title{
Video for teaching purposes
}

\author{
ChrisVassilas \& Luk Ho
}

Over the past 20 years or so, video has become a ubiquitous medium for teaching in psychiatry. It is increasingly being used in both undergraduate and postgraduate teaching. One of the reasons that we wrote this article was that when we wanted to obtain guidance on how to develop the use of video in our own teaching programme, there seemed to be little in the way of concise practical advice available. The focus of the paper will therefore be on the practicalities involved in how feedback from video recordings of interviews can be used to help with the acquisition of clinical skills. We will attempt to give an overview of the variety of different applications for video and give a brief account of the historical development of video feedback in medical training. Appendix 1 is a guide to the practical and technical side of setting up a video recording session. We think that the experiences that we gained while teaching psychiatry in a district general hospital will be useful to all those involved in teaching wherever they are based.

\section{Using video feedback to teach interview skills}

\section{Background}

Traditionally, medical students and junior doctors have been left to their own devices to develop interviewing skills through observation of senior clinicians at ward rounds or clinics and through repeated practice, without much in the way of supervision or feedback. This in itself would be of little importance except that numerous studies have confirmed that these traditional methods do not give doctors the interviewing skills they need. For instance, Maguire \& Rutter (1976) showed that medical students who had trained in the traditional way had serious gaps in their history- taking skills to the extent that many were unable to identify the patient's main problem or clarify the nature of the problem. In addition, the use of lengthy, repetitive closed questions was common. Over 25 years ago, a series of consultations taking place in general practice was recorded on audiotape. Analysis of the tapes revealed that there was considerable scope for improving the quality of these consultations. The Royal College of General Practitioners has subsequently developed methods for feeding back information to doctors about their consultations using video, in order to help them to improve their consulting skills. Analysis of the consultations of trainee general practitioners has now become a routine part of their training.

It could be argued that for psychiatry in particular the traditional training approach is inadequate, since in the absence of any definitive laboratory investigations, the clinical interview remains the cornerstone of the diagnostic process. The 1960s saw the first use of video or teaching purposes in psychiatry, but this was primarily to enable students and trainees to see psychiatric consultations taking place with experienced psychiatrists. In the 1970s, psychiatrists in Manchester began to develop the use of video feedback techniques to teach psychiatric interviewing skills not only to medical students and doctors but to primary care nurses and community workers (Gask, 1998). Just as importantly, they have

Christopher Vassilas is a consultant in old age psychiatry in South Birmingham (The Queen Elizabeth Psychiatric Hospital, Mindelsohn Way, Birmingham B15 2OZ) and Honorary Senior Lecturer at the University of Birmingham. He was until recently the Speciality Tutor in Old Age Psychiatry for East Anglia and the Psychiatric Tutor in the West Suffolk Hospital, where most of the ideas regarding this article were developed. Luk Ho is a specialist registrar in general and old age psychiatry on the East Anglian rotation and is extensively involved in the University of Cambridge undergraduate medical teaching programme (Department of Psychiatry, Box 189, Addenbrooke's Hospital, Hills Road, Cambridge CB2 2QQ). 
also studied the impact of these techniques and accumulated a body of evidence demonstrating that it is possible to produce positive changes in interviewing skills.

Vaughn \& Marks (1976) examined the differences in interviewing skills between medical students who had received video feedback (VT) training compared with a control 'traditional training' (TT) group. Outcome measures used to compare the groups included information elicited, ratings of interview style and counts of classified units of speech (e.g., open-ended and closed questions, facilitation and clarification). VT students did not show superiority in eliciting information but were more likely to avoid closed questions and facilitate the interview.

Even if video feedback improves interviewing skills, much cheaper and simpler methods, such as feedback by audiotape, might be just as effective. Maguire et al (1978), in another study of medical students, found that although differences between the audio- and videotape groups were not statistically significant, they favoured students in the video group. Video feedback appeared to be of particular value in helping the students to take control of the interview (e.g. learning when to interrupt and bring the patient back to the point), avoid unnecessary repetition, ask questions about psychosexual adjustment and detect verbal leads.

In an attempt to find out whether these skills are retained, Maguire et al (1986) reassessed doctors who had received video feedback training during their student days, 5 years before. They found that they maintained their superiority in interviewing skills over their traditionally trained counterparts. Although students given feedback training acquired and retained certain interviewing skills, these had no impact on diagnosis and management.

\section{Teaching the teachers}

Clearly, it is desirable that the teaching of video feedback techniques is as effective as possible. It is possible to teach clinicians how to teach interviewing skills to medical students with only brief training (Naji et al, 1986). Group teaching based on video feedback of the doctor's own consultation significantly improves the ability of general practitioners to teach psychiatric skills (Gask et al, 1991).

The training of those who teach interview skills is of central importance and tutors themselves should consider undertaking a course on how to teach communication skills or how to run video feedback sessions. Tutors and others involved with using these techniques will also find that being aware of the research literature and evidence base for this type of teaching and introducing this into small group sessions can help trainees to learn.

\section{The way forward}

Most psychiatric rotations have added regular video feedback sessions to their training programmes. However, the danger is that such sessions can become informal and unstructured. There is a tendency for the trainee to replay the interview with the trainer occasionally stopping the tape to raise points of discussion. If the patients chosen are already well known to the trainees, the interviews can become displays of their ability to mechanically 'unearth' whatever psychopathology they already know is present, and are therefore of limited use in assessing clinical acumen. In such circumstances, evaluation and discussion tend to focus on the actual information obtained and possible diagnosis and management, without adequate consideration of the techniques used in the interview itself. In a way, this is not surprising as these are the issues to which traditional medical training attaches importance.

\section{Preparation for interviewing}

Video feedback training appears to be most effective when those being trained are provided with a clear model, in which it is made explicit what the component skills are that they are expected to attain (Gask, 1998). Gask (1999)has described the basic communication skills that clinicians need to acquire and her article should be read in conjunction with the present one. It is important that trainees have a clear understanding themselves about which skills are to be learned and evaluated.

Prior to conducting an interview, trainees and medical students should have read and discussed the way in which psychiatric interviews should be conducted. Good accounts are available in standard psychiatric texts.

In addition to the amount of history obtained and diagnostic accuracy, there are other important parameters to be considered in evaluating interviewing skills, which the trainees need to be aware. These include body language, the ability to facilitate the interview, putting the patient at ease, displaying empathy, the recognition of verbal and non-verbal cues and usage of open-ended questions.

Initially, it is useful to have a clear idea of the purpose of the interview and to focus on one aspect (e.g. getting a history of depression or eliciting the first-rank symptoms of schizophrenia), before 
attempting to obtain the entire history and perform a full mental state examination. With psychiatric trainees, we have found it better to use patients that are previously not known to the trainee.

\section{Playing back the videotaped interview and Pendleton's rules}

Playing back interviews in front of a group with a facilitator is an effective and acceptable way of providing feedback - it is also considerably less timeconsuming than one-to-one feedback. However, it is easy to forget how anxiety-provoking it can be for trainees to see themselves on video, particularly in front of a consultant and other trainees. It is important to be aware of this and to control the group sessions effectively. There are techniques that have been devised, originally for use with general practitioners, which aim to reduce any anxiety to a minimum and still allow constructive discussion to take place. These guidelines (Box 1) have become known as 'Pendelton's rules' (Pendleton et al, 1984). What they offer is a safe environment in which learners are able to assess their own performance freely and this is a prerequisite for any constructive learning to take place.

\section{Running a feedback session}

More recently, refinements have been suggested which build on Pendleton's rules but encourage the discussion to be based around the objectives that the trainee has set him- or herself. Kurtz et al (1998) have written a book which outlines this method based on teaching programmes in the Medical Faculty at the University of Calgary, Canada and in postgraduate general practice in East Anglia. This is described as a problem-based approach and medical educationalists claim that using this

\section{Box 1. Pendleton's rules}

Clarify matters of fact briefly

Interviewer says what he or she thinks has been done well

Rest of the group then say what they think has been done well

Interviewer says what he or she thinks could have been done differently

The rest of the group then say what they think could have been done differently method is more likely to motivate adults, in particular, to learn. Initially grasping this different way of working can be more difficult for trainers than using the traditional didactic approach, but research evidence has confirmed its superiority. But what does this mean in practice? Box 2 offers a summary of what such an approach might be, adapted from Kurtz et al (1998). A structured approach ensures that both trainees and trainers know what is expected of them during the feedback sessions.

Our feedback sessions take place in a group setting with a senior psychiatrist and the trainees. It is useful for the trainee who made the video to make the first comments about his or her performance, raising any problems experienced and suggesting the areas with which he or she would like help. Trainers should encourage the trainee to problem solve and also get the group involved in suggesting solutions. Initially, the group may find this difficult and trainers must make a special effort to encourage them. It is important that feedback is balanced and that good points as well as areas for improvement are discussed. Another important principle about feedback is that it should be descriptive. This means that rather than statements such as "That was really good", specific comments are made on performance which are non-judgemental, for example, "You started with open

Box 2. Problem-based analysis (adapted from Kurtz et al, 1998)

Start with the trainee's agenda

Look at the outcomes that the interview is trying to achieve

Encourage self-assessment and self-problemsolving first

Involve the whole group in problemsolving

Used descriptive feedback

Feedback should be balanced (what worked and what could be done differently)

Suggest alternatives

Rehearse suggestions through role-play

Be supportive

The interview is a valuable tool for the whole group

Introduce concepts, principles and research evidence as opportunities arise

At the end, structure and summarise what has been learnt 
questions and then moved on to closed questions". If there are issues about how the interview could have been improved, the group should be invited to make suggestions. Role-play can be introduced into the session to explore particular interview skills for instance, phrasing, how to elicit particular symptoms or what to say at a difficult point in the interview. The tutor should take opportunities as they arise to introduce concepts and research evidence - brief didactic teaching at appropriate points in the discussion effectively complements learning from group work.

At the end, it is the trainer's responsibility to summarise the session in a structured way. These steps will help to minimise stress and ensure the focus of feedback is not on the trainee's shortcomings. When discussing areas of difficulty, it is essential to make recommendations as to how these tasks might have been achieved. The advantage of video is of course that if a particular point is raised in the discussion the video can be rewound and the interview looked at again.

\section{Rating the interview}

An integral part of Kurtz et al's (1998) approach is to check any interviews against a checklist they entitle their list 'The Calgary-Cambridge Observation Guide' and it consists of two parts. Part one, which covers basic interview skills, has five sections (Box 3), which are further subdivided to cover the skills required in developing interview skills. There is space for comments alongside each section. Together with part two of the guide, this represents a comprehensive summary of the communication skills that clinicians are expected to acquire - it is intended to be used for undergraduate teaching, speciality training and continuing medical education.

The authors acknowledge that it is necessary to alter the format of the guide for different groups of learners. We have indicated in Box 3 some of the skills that we have found to be useful for psychiatric trainees to develop in our training programme and these are placed in italics underneath the relevant heading. In addition, trainees will inevitably ask for feedback on eliciting psychopathology and on their diagnostic skills, and there must be room for discussion of these points in feedback sessions.

How should these lists be used? They are not supposed to be a checklist in the normal sense of the word, with trainees having to complete all the tasks listed. Instead, they act as a framework for sessions so that trainees can write down comments in a structured way (again using descriptive, nonjudgemental comments) as the video is played to them. At the end of the session the list can help the trainer with his or her summary of the session, so that he or she can document the skills that have been discussed.

\section{Training needs of different groups}

The objectives of each interview need to be tailored to the needs of each trainee: a psychiatric trainee preparing for Part II of the MRCPsych Examination will have different needs from a general practice trainee spending 6 months in a psychiatric senior house officer post.

We have also asked the medical students who have been attached to us to record a patient interview and have found this a good way to teach clinical skills. The aims in undergraduate teaching are more modest than for postgraduates, with the emphasis on establishing a rapport with patients and basic history-taking skills. Of course, undergraduates need more supervision in recording interviews and guidance about what is required. Points raised

Box 3. Aspects of the interview to be rated

Initiating the interview

General greeting

Introduction of self and explanation of session

Opening question

Setting the agenda

Gathering information

Question style, using open questions and moving to closed questions appropriately

Facilitation and clarification of patient's responses

Listening to patient, using easily understood questions

Checking patient's understanding of questions

Establishing dates

Keeping control of the interview

Building the relationship

Non-verbal behaviour, posture, eye-contact

Empathy and support when sensitive topics arise

Explanation and planning

Closing the session

Summarising problems and giving explanation if required

Terminating session appropriately 
during the interview can be used as the basis for further discussion in the rest of the undergraduate training programme.

\section{Other uses of video}

\section{Collecting a library of tapes}

Medical educators term the process by which trainers and other doctors demonstrate skills that are then observed by others as 'modelling'. There is no doubt that this is valuable tool in conjunction with other methods of learning. It is useful to build up a library of pre-recorded interviews by experienced trainers. Examples of topics include: the demonstration of 'classic' histories and psychopathological signs; how to deal with uncooperative patients; performing an assessment of cognitive function; and the discussion of sensitive subjects (e.g. breaking bad news). Of course, a variety of commercial tapes are now available which cover most of these areas.

Medical students often have preconceived ideas of what psychiatry is like and who psychiatric patients are - the use of videotaped interviews can provide an useful way of breaking down these stereotypes, as well as being an introduction to the psychiatric interview - for instance, demonstrating how a psychiatric interview can be used in medical patients.

Seeing the trainer interviewing a patient is also useful at the start of a series of sessions where trainees will be using video feedback.

\section{Using video in examinations}

The Royal College of Psychiatrists has recently reviewed the Membership exam and objective, structured clinical examinations (OSCEs) will be introduced in spring 2003. These are widely used in North America and consist of several stations, each presenting a different clinical scenario. Examinees rotate around each station after a predetermined period of time. One possibility might be to have a station consisting of a short video recording of an interview and asking examinees to comment on the psychopathology.

It has been suggested that examinees could be videoed interviewing patients - the interview process is less likely to be distorted by the physical presence of the examiners and re-runs of the tapes could be used to sort out scoring discrepancies (Connolly \& Bird, 1977). The OSCE format would be well suited to this process and would allow for the use of simulated patients.
Yet another use of video is that introduced by The Royal College of General Practitioners. General practice trainees video tape their real-life consultations and submit them as part of the Membership exam.

\section{Multimedia computer-based learning}

The power of modern desktop computers is now such that they allow the user to make full use of multimedia learning. Video recordings of patients or simulated patients can be made and incorporated into a learning package that is then played back by the user on his or her own computer. The key to this approach to learning is that the learner interacts with the computer package rather than being a passive recipient of information, for example, by answering questions about psychopathology or being asked to make a diagnosis. To date, as Williams et al (1999) point out, few packages have been formally peer-reviewed or evaluated in terms of effectiveness for teaching purposes. However, there is an enormous potential for developing undergraduate and postgraduate teaching as well as continuing professional development along these lines.

\section{Other uses}

In psychiatric case conferences, videotapes of interviews with patients are increasingly used instead of the rather intimidating 'grand round', where patients were often interviewed in front of an audience of scores of people. Trainees have in the past found it useful to record mock exams or even mock interviews for prospective jobs. Playing back these recordings can provide useful feedback.

Increasingly, video recordings are being produced in the area of patient and carer education, and organisations dealing with particular psychiatric conditions are involved in producing educational videos. For instance, a variety of videos are available aimed at workers and carers dealing with dementia and schizophrenia (see Appendix 2).

\section{Conclusions}

Video is a powerful tool in psychiatric training and education. It will be increasingly used in assessing the skills of undergraduates and postgraduates and for examination purposes. 
With a little thought and planning, most psychiatric rotations should be able to incorporate video into their regular teaching programmes. Main areas for use will be a library of videotapes and video feedback for training purposes.

There is now a body of research evidence to support the superiority of video feedback over traditional teaching methods. In order to be effective, video feedback sessions need to be standardised, and psychiatrists training others using video will themselves benefit from further training. We believe the potential in this area has yet to be fully realised.

\section{Appendix 1}

\section{The practicalities of video recording}

\section{Equipment}

Finding suitable equipment is a potential stumbling block. Some departments in larger hospitals may be fortunate enough to have access to a dedicated, fully equipped audio-visual studio. Often, a technician who has the required expertise can help to make certain that high quality recordings are obtained. With specialist equipment, tapes can even be edited to ensure that relevant segments of the interview are highlighted or that the same interview is recorded from several points of view.

However, those psychiatrists working in smaller hospitals may not have the luxury of such a setup. Our experience has been that with the amateur equipment now available in high street stores, high quality recordings can still be made which can be used for video feedback sessions or to establish a library of tapes as we outline below. There are several formats of video equipment available (Box 4): we have successfully used a VHS-C camcorder. Modern camcorders are compact video cameras that have room for a small cassette containing videotape - most can play back their recordings via a cable connecting the camcorder to a television monitor. Technology is changing very fast in this area, so there is little point in giving detailed advice on equipment - instead we have tried to give some general advice. Further information on which specific equipment ought to be purchased should be sought from colleagues with some technical knowledge, consumer magazines such as Which? magazine and from specialist stores.

Box 5 lists the equipment requirements for a basic video recording session. When buying equipment, remember that for teaching purposes tapes are going to played and replayed in order to determine what has happened during the interview. The sound quality of the recording is therefore of paramount importance. Camcorders vary in how well they record sound so ask to see the equipment in use before agreeing to purchase. We would stress that using an external microphone and a sturdy tripod will make a huge difference to the quality of recordings that can be obtained.

It is essential if trainees do not have the benefit of a 'professional' set-up that they are thoroughly familiar with the equipment before they attempt to record any interviews. Ideally, familiarisation with the equipment and room to be used for video recordings should take place at an induction session. Medical students will probably need supervision for the recording sessions. Instructions on how to set up the equipment and on which leads go where can be summarised on a side of A4 paper and copies made and kept with the equipment.

Trainers should aim to produce a situation in which interviews take place without the interviewer or interviewee being distracted by the technical process of videoing the proceedings. Our experience has been that with some practice beforehand this is an achievable goal.

\section{Patient consent}

Standardised consent forms should be used for prospective interviewees. In many cases, interviews are erased after viewing and patients need to be aware of this.

Box 4. Formats of video-tape commonly used

VHS: this is no longer common and the equipment is bulky; it is the format you would get on a video borrowed from the local video store

There are a variety of popular, smaller tape formats now in use, including:

- VHS-C (little tapes that, with an adapter, play back on a VHS video recorder)

- $8 \mathrm{~mm}$

- digital format: this is the newest format, offering very high quality; prices are higher than other formats - although as with all new, popular technologies prices will probably begin to fall

High-resolution versions of the VHS and 8 $\mathrm{mm}$ formats, called SuperVHS (or SVHS), S-VHS-C and Hi8mm 
The General Medical Council (GMC) and the Royal College of Psychiatrists (RCPsych) have published guidelines on making and using visual and audio recordings of patients (GMC, 1997; RCPsych, 1998). These discuss the ethical issues and issues of confidentiality which arise from using these recordings for training and assessment purposes. Any doctor intending to make recordings of patents should acquaint themselves with these guidelines.

Box 5. Equipment requirements for making videos of interviews

Well-ventilated room. The room should be free from noise and the possibility of interruption and should be large enough to allow the camera to record both interviewer and interviewee

Two comfortable chairs

Video camcorder - make sure that it has the ability to accept an external microphone

External microphone and microphone stand (these are essential if using an 'amateur' camcorder). There should be somewhere to place the microphone equidistant between the interviewer and interviewee

Sturdy tripod (again essential) - there is no point in getting a flimsy tripod

Television monitor

Most camcorders can be directly connected to a television monitor so that tapes can be played back without the necessity of a separate video player. However, check before purchasing as some camcorders may require a special type of connector on your television monitor and some monitors may not have this

Blank tape for recording

Fully charged battery packs if required for any of the equipment

Relevant leads to connect the equipment together

Written informed consent from patient using a standard form

Copies of instructions for setting up the equipment

\section{In addition}

There should be provision for the secure storage of equipment

A VHS video recorder will allow playback of pre recorded material

\section{Appendix 2}

\section{Where to find video recordings}

The British Universities Film and Video Council (BUFVC) has an on-line database (AVANCE) which gives details of a large number of video recordings on psychiatric topics at www.bufvc.ac.uk.

The Alzheimer's Disease Society (Gordon House, 10 Greencoat Place, London, SW1P 1PH) has a selection of videos available dealing with dementia.

The National Schizophrenia Fellowship (28 Castle St, Kingston upon Thames, Surrey KT1 1SS) has a large library of videotapes available for hire for a small fee. The tapes are likely to be of value to carers, professionals and also to sufferers.

Another source of videotapes is the pharmaceutical companies, many of which have a selection of recordings available that are useful for mental health professionals and also patients.

\section{References}

Byrne, P. S. \& Long, B. E. L. (1984) Doctors Talking to Patients. Exeter: The Royal College of General Practitioners.

Connolly, J. \& Bird, J. (1977) Videotape in teaching and examining clinical skills: a short case format. Medical Education, 11, 271-275

Gask L., Goldberg D., Boardman J., et al (1991) Training general practitioners to teach psychiatric interviewing skills: an evaluation of group training. Medical Education, 25, $444-451$.

- (1998) Small group interactive techniques utilizing video feedback. International Journal of Psychiatry in Medicine, 28, 97-113.

- (1999) Acquisition of clinical skills. Advances in Psychiatric Treatment, 5, 311-316.

General Medical Council (1997) Making and using visual and audio recordings of patients. www.gmc-uk.org

Kurtz, S., Silverman, J. \& Draper, J. (1998) Teaching and Learning Communication Skills in Medicine. Abingdon: Radcliffe Medical Press.

Maguire, P. \& Rutter, D. (1976) History taking for medical students. 1. Deficiencies in performance. Lancet, 2, 556558.

—, Roe, P., Goldberg, D., et al (1978) The value of feedback in teaching interviewing skills to medical students. Psychological Medicine, 8, 695-704.

-, Fairbairn, S. \& Fletcher, C. (1986) Consultation skills of young doctors: I - benefits of feedback training in interviewing as students persist. British Medical Journal, 292, 1573-1576.

Naji, S. A., Maguire, G. P., Fairburn, S. A., et al (1986) Training clinical teachers to teach interviewing skills to medical students. Medical Education, 20, 140-147.

Pendleton, D., Schofield, T., Tate, P., et al (1984) A method for giving feedback. In The Consultation: An Approach to Learning and Teaching, pp. 68-71. Oxford: Oxford University Press.

Royal College of Psychiatrists (1998) Guidance for Videotaping. Council Report CR65. London: Royal College of Psychiatrists. 
Vaughan, M. \& Marks, J. N. (1976) Teaching interviewing skills to medical students: a comparison of two methods. Medical Education, 10, 170-175.

Williams, C. J., Aubin, S., Harkin, P. J. R., et al (1999) Anxiety

Disorders. Leeds: University of Leeds Innovations Service.

\section{Multiple choice questions}

1. Studies which examined the differences in interviewing skills between medical students who received video feedback training (VT) compared with a control 'traditional' training (TT) group revealed the following:

a VT students showed superiority in eliciting information

b VT students were more likely to avoid closed questions

c outcome measures used included counts of classified units of speech

d there were significant differences between audio- and videotape groups

e video training failed to help students to take control of interviews.

2. In follow-up studies:

a Maguire et al reassessed students 5 years later

b doctors who received video feedback training during their student days maintained their superiority in interview skills when reassessed

c feedback training had a significant impact on diagnosis and management

d traditionally trained students showed improved coverage of psychosocial problems with time

e doctors did particularly well with physically ill students.

3. Important parameters to be considered in evaluating interviewing skills about which the trainees need to be aware include:
a body language
b ability to facilitate the interview
c display of empathy
d recognition of verbal rather than non-verbal cues
e avoidance of open-ended questions.

4. During video feedback sessions:

a the trainee who made the video should avoid making the first comments about his or her performance

b trainers should encourage the trainee to problem solve and also get the group involved in suggesting solutions

c it is important that feedback is balanced (good points and bad points) and based on description

d when discussing areas of difficulty, it is essential to recommend how tasks might have been achieved

e phrasing can be rehearsed through role-play.

5. To make a video of an interview:

a a spacious well-ventilated room is needed

b written informed consent from the patient is not required

c only fully dedicated and well-equipped audio-visual studios should be used

$d$ trainees should be instructed on the operation of the equipment as part of their induction

e sound quality is not an important consideration.

MCQ answers

\begin{tabular}{|c|c|c|c|c|}
\hline 1 & 2 & 3 & 4 & 5 \\
\hline F & a $T$ & a $\mathrm{T}$ & a $\mathrm{F}$ & a 1 \\
\hline$T$ & b $T$ & b $T$ & b $\mathbf{T}$ & b \\
\hline $\mathrm{T}$ & c $F$ & c $T$ & c 1 & c $F$ \\
\hline $\mathbf{F}$ & d $F$ & d F & d $T$ & $\mathrm{~d}$ \\
\hline F & e $F$ & e $F$ & e 1 & e \\
\hline
\end{tabular}

